\title{
Funktionelle Glasmembranen für elektrochemische Sensoren in Dünnfilmtechnik
}

\author{
F. Gerlach ${ }^{1}, K$. Ahlborn $^{1)}$, H. Iken ${ }^{2)}$, T. Bronder ${ }^{2}$, M.J. Schöning ${ }^{2)}$, W. Vonau ${ }^{1)}$ \\ 1) Kurt-Schwabe-Institut für Mess- und Sensortechnik e.V., Meinsberg, Waldheim, Deutschland \\ 2) Institut für Nano- und Biotechnologien, FH Aachen \\ University of Applied Sciences, Jülich, Deutschland \\ gerlach@ksi-meinsberg.de
}

\section{Zusammenfassung}

Ziel des Projektes ist die Entwicklung geeigneter Dünnfilmtechnologien zur Herstellung von neuartigen ionensensitiven und redoxsensitiven Strukturen auf der Basis silikatischer Gläser sowie deren Fertigung und Charakterisierung. In diesem Beitrag werden Vorteile, Möglichkeiten und Grenzen der Erzeugung von silikatischen Schichtstrukturen mittels der gepulsten Laserablation (PLD) [1,2] aufgezeigt. Für die Charakterisierung der sensitiven Schichtstrukturen wurden verschiedene röntgenbasierte Verfahren, wie z.B. Energiedispersive Röntgenspektroskopie (EDX), Röntgenfluoreszenzanalyse ( $\mu$-RFA) und Röntgendiffraktometrie (XRD) [3] herangezogen.

Keywords: PLD-Technik, $\mu-R F A$, sensitive Glasschichten,Schichtcharakterisierung

\section{Einleitung}

PLD ist ein Verfahren zur stöchiometrischen Übertragung von komplexen Materialkompositionen, u.a. Glasstrukturen, von einem Target auf Substratmaterialien, insbesondere Glasund Keramiksubstrate mit einem angepassten Ausdehnungskoeffizienten.

Elektrochemische Sensorstrukturen bestehen i.d.R. aus einer edelmetallbasierten Ableitelektrode mit darunterliegender Haftschicht, einer gemischtleitendenden Zwischenschicht zur Gewährleistung einer guten Potenzialstabilität und der eigentlichen sensitiven Schicht. Durch die Kombination verschiedener Vakuumbeschichtungsverfahren einschließlich der Siebdrucktechnik sind optimierte Lösungsansätze sowohl hinsichtlich technischer Parameter als auch ökonomischer Kennziffern möglich.

Mittels gepulster Laserablation gelingt es, komplexe und glastechnisch schwer verarbeitbare, d.h. sich an der Entmischungsgrenze befindliche Glassysteme, zu dichten Schichten auf planaren Substraten abzuscheiden.

\section{Experimentelles}

\section{Elektrodenherstellung}

Wesentlicher Bestandteil potenziometrischer elektrochemischer Sensoren ist die sensitive Membran der Indikatorelektrode. Hinsichtlich des Leitungsmechanismus innerhalb der Membranen wird zwischen Elektronen- und lonenleitung unterschieden. So sind für die Messung der Aktivität von Wasserstoff- und einer Reihe von Alkaliionen ionenleitende Silikatgläser bekannt [4]; zur Bestimmung des Redoxpotenzials nutzt man Messfühler auf der Basis von elektronisch leitfähigen Silikatgläsern mit Metalloxiden unterschiedlicher Wertigkeit [5]. Die Performance der glasbasierten Elektroden zur Bestimmung des Redoxpotenzials orientiert sich an den für diesen Zweck weltweit etablierten Edelmetallelektroden, wobei insbesondere im Vergleich zum Platin der Einfluss von Kontaktgiften und der des pH-Wertes an den Gläsern deutlich geringer ist [6].

Für Untersuchungen und Analysen an $\mathrm{pH}-$ sensitiven Gläsern wurden die in Tabelle 1 aufgeführten Kompositionen ausgewählt. 
Tab. 1: Glaskompositionen $\mathrm{pH}$-sensitiver Gläser

\begin{tabular}{|c|ccccccc|}
\hline Glas & A & V10 & C & V11 & V27 & V2A & DS1 \\
\hline $\mathrm{Si}$ & $\mathrm{X}$ & $\mathrm{X}$ & $\mathrm{X}$ & $\mathrm{X}$ & $\mathrm{X}$ & $\mathrm{X}$ & $\mathrm{X}$ \\
$\mathrm{Li}$ & & & $\mathrm{X}$ & $\mathrm{X}$ & $\mathrm{X}$ & $\mathrm{X}$ & \\
$\mathrm{Na}$ & $\mathrm{X}$ & $\mathrm{X}$ & & & & & $\mathrm{X}$ \\
$\mathrm{La}$ & & & & & & $\mathrm{X}$ & \\
$\mathrm{Mn}$ & & & & $\mathrm{X}$ & $\mathrm{X}$ & $\mathrm{X}$ & \\
$\mathrm{Nb}$ & & & & & & $\mathrm{X}$ & \\
$\mathrm{Nd}$ & & & & & & $\mathrm{C}$ & \\
$\mathrm{Ca}$ & $\mathrm{X}$ & $\mathrm{X}$ & & & & & \\
$\mathrm{Ba}$ & & & $\mathrm{X}$ & $\mathrm{X}$ & $\mathrm{X}$ & & \\
$\mathrm{Mg}$ & & & & & & & $\mathrm{X}$ \\
$\mathrm{Sn}$ & & & $\mathrm{X}$ & & & & \\
$\mathrm{Ti}$ & & & $\mathrm{X}$ & $\mathrm{X}$ & $\mathrm{X}$ & & \\
$\mathrm{Pb}$ & & & $\mathrm{X}$ & & & & \\
$\mathrm{Cs}$ & & & $\mathrm{X}$ & & & & \\
\hline
\end{tabular}

Adäquat geschah dies auch für die redoxsensitiven Gläser mit dem Ziel, ein Optimum hinsichtlich Verarbeitbarkeit und Messverhalten zu erzielen. Die verwendeten Kompositionen sind in Tabelle 2 aufgeführt.

Tab. 2: Glaskompositionen redoxsensitiver Gläser

\begin{tabular}{|c|ccccccc|}
\hline Glas & F7 & F17 & F23 & F27 & F28 & T10 & GL \\
\hline $\mathrm{Si}$ & $\mathrm{X}$ & $\mathrm{X}$ & $\mathrm{X}$ & $\mathrm{X}$ & $\mathrm{X}$ & $\mathrm{X}$ & $\mathrm{X}$ \\
$\mathrm{Li}$ & $\mathrm{X}$ & $\mathrm{X}$ & $\mathrm{X}$ & $\mathrm{X}$ & $\mathrm{X}$ & & \\
$\mathrm{Na}$ & $\mathrm{X}$ & & $\mathrm{X}$ & $\mathrm{X}$ & $\mathrm{X}$ & $\mathrm{X}$ & $\mathrm{X}$ \\
$\mathrm{Fe}$ & $\mathrm{X}$ & $\mathrm{X}$ & $\mathrm{X}$ & $\mathrm{X}$ & $\mathrm{X}$ & & $\mathrm{X}$ \\
$\mathrm{K}$ & & & & $\mathrm{X}$ & & $\mathrm{X}$ & \\
$\mathrm{Ti}$ & & & & & & $\mathrm{X}$ & \\
$\mathrm{Nb}$ & & & & & & $\mathrm{X}$ & \\
$\mathrm{Ca}$ & & $\mathrm{X}$ & $\mathrm{X}$ & $\mathrm{X}$ & & & \\
$\mathrm{Al}$ & & & & & & & $\mathrm{X}$ \\
\hline
\end{tabular}

Die zu untersuchenden Glastargets wurden in einer Hochtemperaturkammer erschmolzen und in einem Gießprozess in speziell vorgeheizten Graphitformen direkt aus der Glasschmelze gewonnen. Diese Herstellungsmethode führt zu homogenen und zugleich amorphen Targetmaterialien mit definierten Geometrien, die in unterschiedlichen PLDAnlagen Verwendung fanden. Hergestellt wurden Glaszylinder mit $18 \mathrm{~mm}$ Durchmesser und zylindrische Scheibentargets von $28 \mathrm{~mm}$ Durchmesser (Abbildung 1), die mittels einer Präzisionstrennsäge (Accutom - 50 der $\mathrm{Fa}$. Struers) in Scheiben mit einer Dicke von $5 \mathrm{~mm}$ vereinzelt wurden.

Um die Gläser in konventioneller Art und Weise glasbläserisch weiterverarbeiten zu können, kamen in Graphitformen gegossene Glasstäbe zum Einsatz.
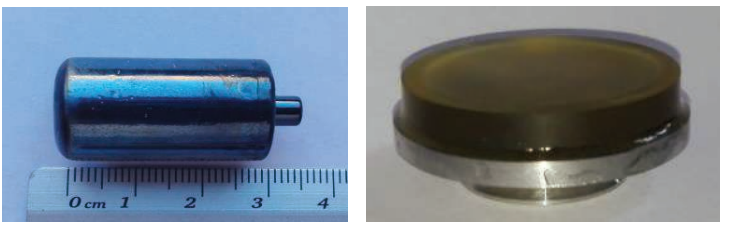

Abb. 1: hergestellte Glastargets in unterschiedlicher geometrischer Ausführung

Die Herstellung der dünnen Schichten erfolgte mit Sputter- und Pulsed Laser Deposition (PLD)-Methoden. Hierzu wurde eine kombinierte Beschichtungsanlage "CREAMET 500 PLD S2" der Fa. Creavac (Abbildung 2) verwendet, welche beide Beschichtungsmethoden und das Substrat- und Maskenhandling zur Verfügung stellt, ohne das Vakuum während des Beschichtungsprozesses unterbrechen zu müssen.

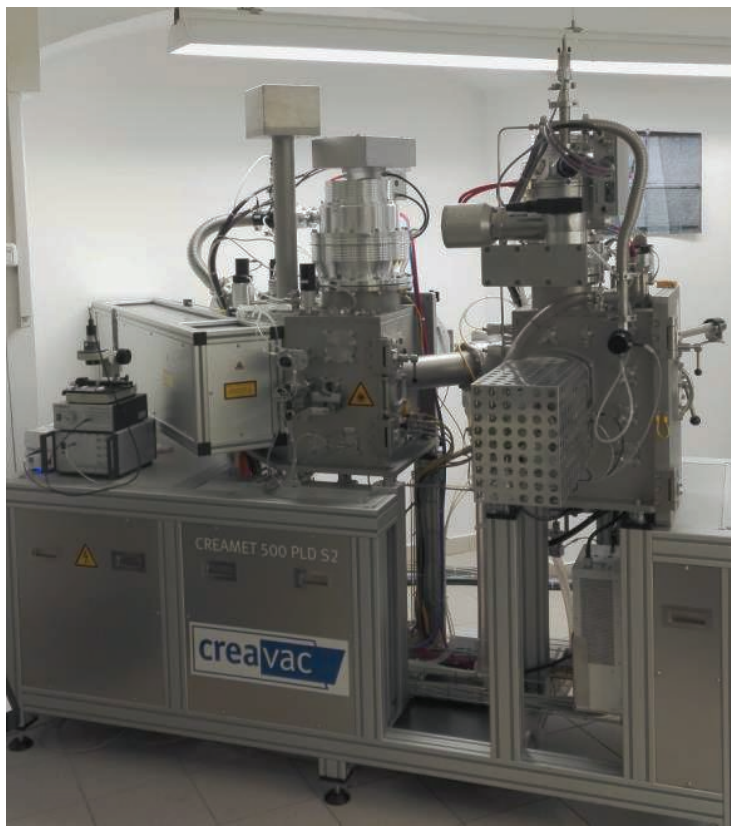

Abb. 2: Kombinierte Beschichtungsanlage CREAMET 500 PLD S2 der Fa. Creavac GmbH, Dresden, Sputterkammer, Masken- und Substrathandler und PLD-Kammer

Mit dem integrierten Substrathandler- und Wechselmaskensystem können 2 Sputtertargets, 6 PLD-Targets und insgesamt 5 Wechselmasken für die Prozesse verwendet und kombiniert werden. Als Substrate kamen vorgereinigte, rechteckige Glasscheiben, bestehend aus Natron-Kalk-Glas in einer Größe von $50 \mathrm{~mm} \times 15 \mathrm{~mm}$ und einer Dicke von $1 \mathrm{~mm}$, zur Anwendung. Diese wurden mit dem vorangestellten Plasmaprozess bei einem Kammerdruck von $3,0 * 10^{-2}$ mbar unter Argon- 
atmosphäre vorbehandelt. Der Kammerdruck für die nachfolgenden Sputterprozesse der Haftschicht ( $\mathrm{Ti})$ und der elektrisch leitfähigen

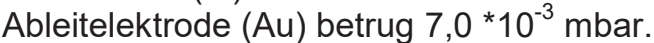

Nach den Sputterprozessen wurden die beschichteten Substrate ausgeschleust und in einer sogenannten "Load-Lock-Box" die Masken gewechselt. Zur Glasplasmaerzeugung diente eine KrF Excimer Laser Quelle (ComPexPro 110 der Fa. Coherent) mit einer Wellenlänge von $248 \mathrm{~nm}$ und einer Fluenz von $5.6 \mathrm{~J} / \mathrm{cm}^{2}$ bei Pulslängen von $20 \mathrm{~ns}$ und einer Pulsfrequenz von $10 \mathrm{~Hz}$. Eine Laserleistungsmessung vor und nach dem Beschichtungsprozess in Verbindung mit einer periodischen Reinigung des Lasereintrittfensters gewährleistete langzeitstabile und reproduzierbare Randbedingungen. Die PLD-Beschichtung erfolgte bei einem Kammerdruck von 3,1 *10-7 mbar in $\mathrm{N}_{2}$ - und $\mathrm{O}_{2}$-Atmosphäre bei Ablationszeiten für die sensitiven Schichten von $10 \mathrm{~min}$ bis 60 min und einer senkrechten Positionierung der Substrate zur Plasmakeule (OnAxis-PLD).

Die Maskierung (Abbildung 3) erfolgte mittels Siebdrucktechnik. Somit konnten reproduzierbare Bedingungen für die analytischen und elektrochemischen Untersuchungen geschaffen werden.

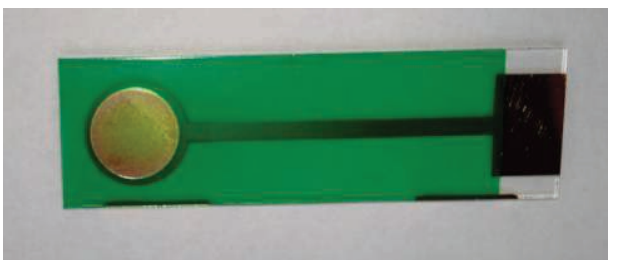

Abb. 3: mittels Siebdrucktechnik maskierte Dünnschichtelektrode auf Glassubstraten

\section{Ergebnisse}

\section{Morphologie}

Erste Untersuchungen der Glasschichten fanden zur Oberflächenmorphologie statt. Hierzu kam ein Digitales Rastermikroskop (REM) Helios 660 der Fa. FEI zum Einsatz. Durch die Verwendung einer Feldemissionskathode konnte bei niedrigen Beschleunigungsspannungen gearbeitet und auf eine zusätzliche Beschichtung der Glasoberflächen verzichtet werden. Die geringen Beschleunigungsspannungen haben darüber hinaus den Vorteil, dass die bildgebenden Informationen nur aus sehr oberflächennahen Schichten erhalten wurden.
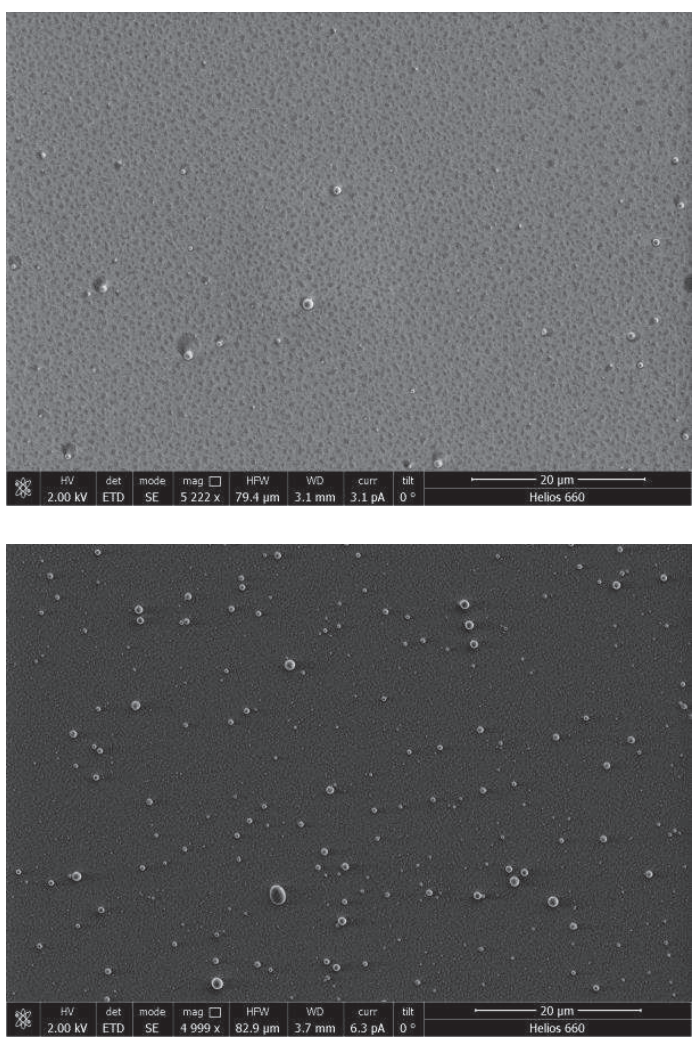

Abb. 4: REM-Bilder von $\mathrm{pH}$-sensitiven Glasschichten (o.: V27 / u.: V10)
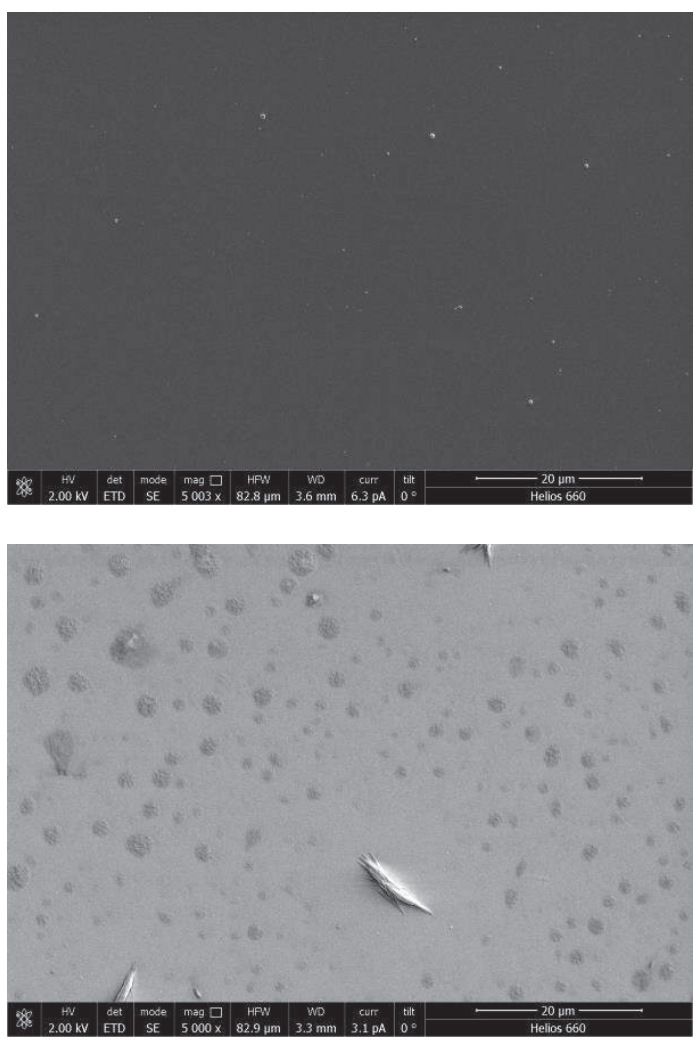

Abb. 5: REM-Bilder von redoxsensitiven Glasschichten (o.: F17 / u.: F28) 

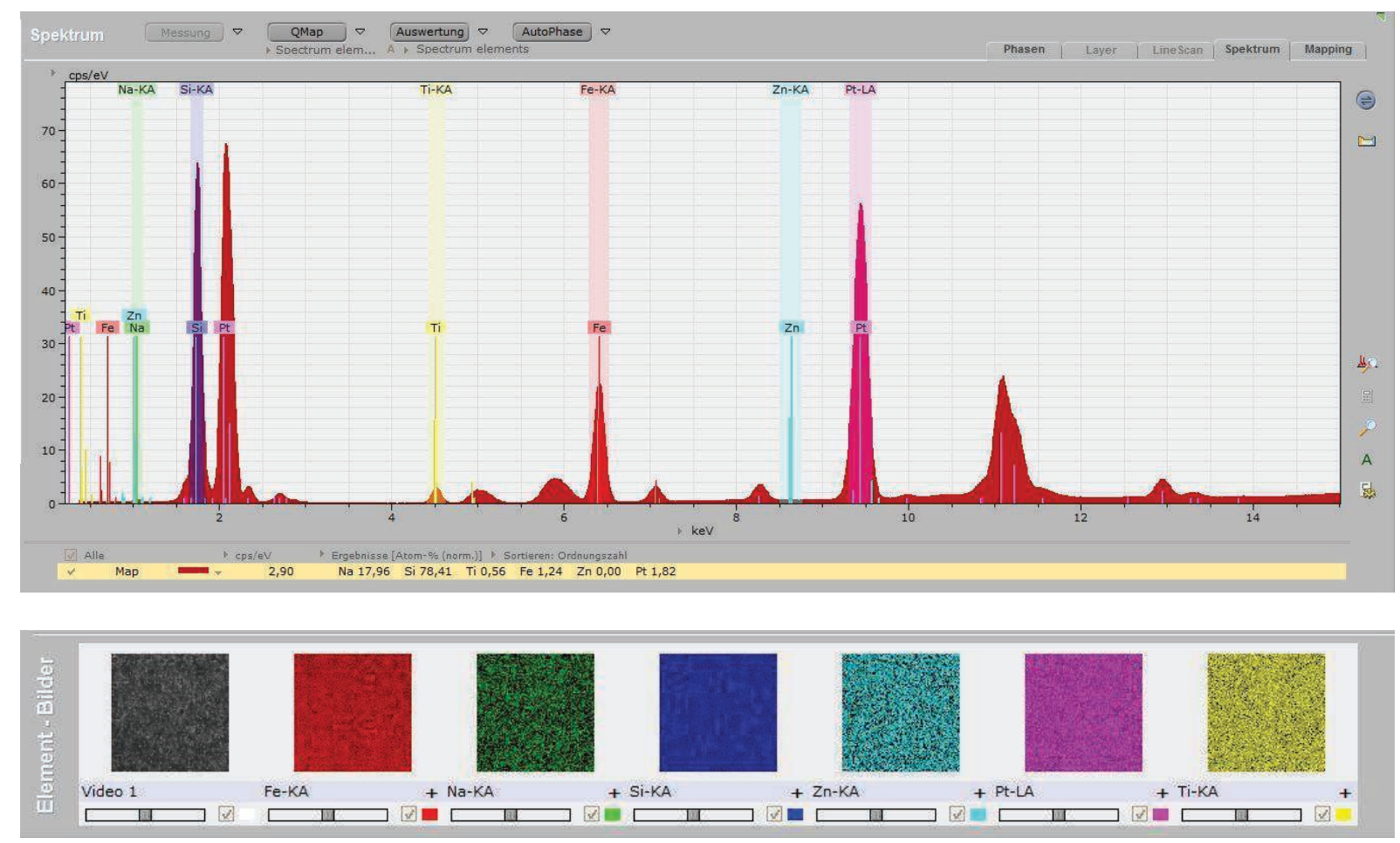

Abb. 6: Spektrum und Elementverteilungsbilder einer mittels PLD-Technologie aufgebrachten redoxsensitiven Glasschicht (F28)

Die pH-sensitiven Schichten (Abbildung 4) zeigen homogene, dichte Schichten mit einer unterschiedlichen Ausbildung von Mikrostrukturen und „Droplets“, welche bis zu einer gewissen Größe und Anzahl die sensitiven Eigenschaften nicht nachteilig beeinflussen. Für die Redoxgläser zeichnen sich ähnliche Schichteigenschaften ab, wobei vereinzelt die Bildung von Siliziumwhiskern auftrat (Abbildung 5 / u.: F28).

\section{$\mu$-RFA}

Die für jedes erschmolzene Glas durchgeführte Mikroröntgenfloureszensanalyse ( $\mu$-RFA) verfolgte das Ziel, die Verteilung der Glasbestandteile zu analysieren. Für die Untersuchungen stand das System M4 Tornado der Firma Bruker Nano GmbH (Berlin) zur Verfügung. Die Abbildung 6 zeigt beispielhaft das aufgenommene Spektrum und die Elementverteilung der Glasbestandteile. Eine qualifizierte quantitative Analyse ist auf Grund der Schichtstruktur und der großen Eindringtiefe des materialanregenden Röntgenstrahls nicht sinnvoll.

\section{XRD}

Die Aufnahmen von Röntgendiffraktogrammen dünner Schichten wurden mit einem speziellen "General Area Detection Diffraction Systems" (GADDS) an einem D8 Discover High Resolution Diffractometer (Fa. Bruker AXS $\mathrm{GmbH}$,
Karlsruhe) durchgeführt. Diese Konfiguration ermöglicht es, die Detektionsgrenze für kristalline Phasen wesentlich zu verringern. In Abbildung 7 ist ein Röntgendiffraktogramm einer sehr dünnen Schicht aus pH-sensitiven Glas dargestellt. Charakteristisch ist der sogenannte "Glasberg" ohne nennenswerte Peaks, welcher auf die amorphe Struktur der Glasschicht hinweist.

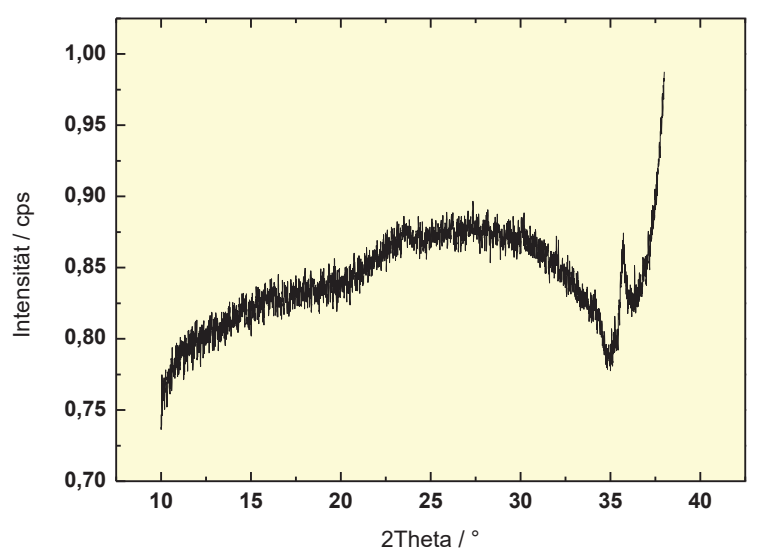

Abb. 7: Diffraktogramm einer dünnen Redoxglasschicht (3 $\mu \mathrm{m})$ 


\section{EDX}

Die Untersuchungen mittels energiedispersiver Röntgenspektroskopie (EDX) sind mit den gleichen Problemen belegt, wie im Abschnitt
Helios 660 der Fa. FEl lassen sich sehr gut ausreichende Signalqualitäten bei unterschiedlichen Beschleunigungsspannungen erzielen. Damit sind die Anregungsvolumina variabel
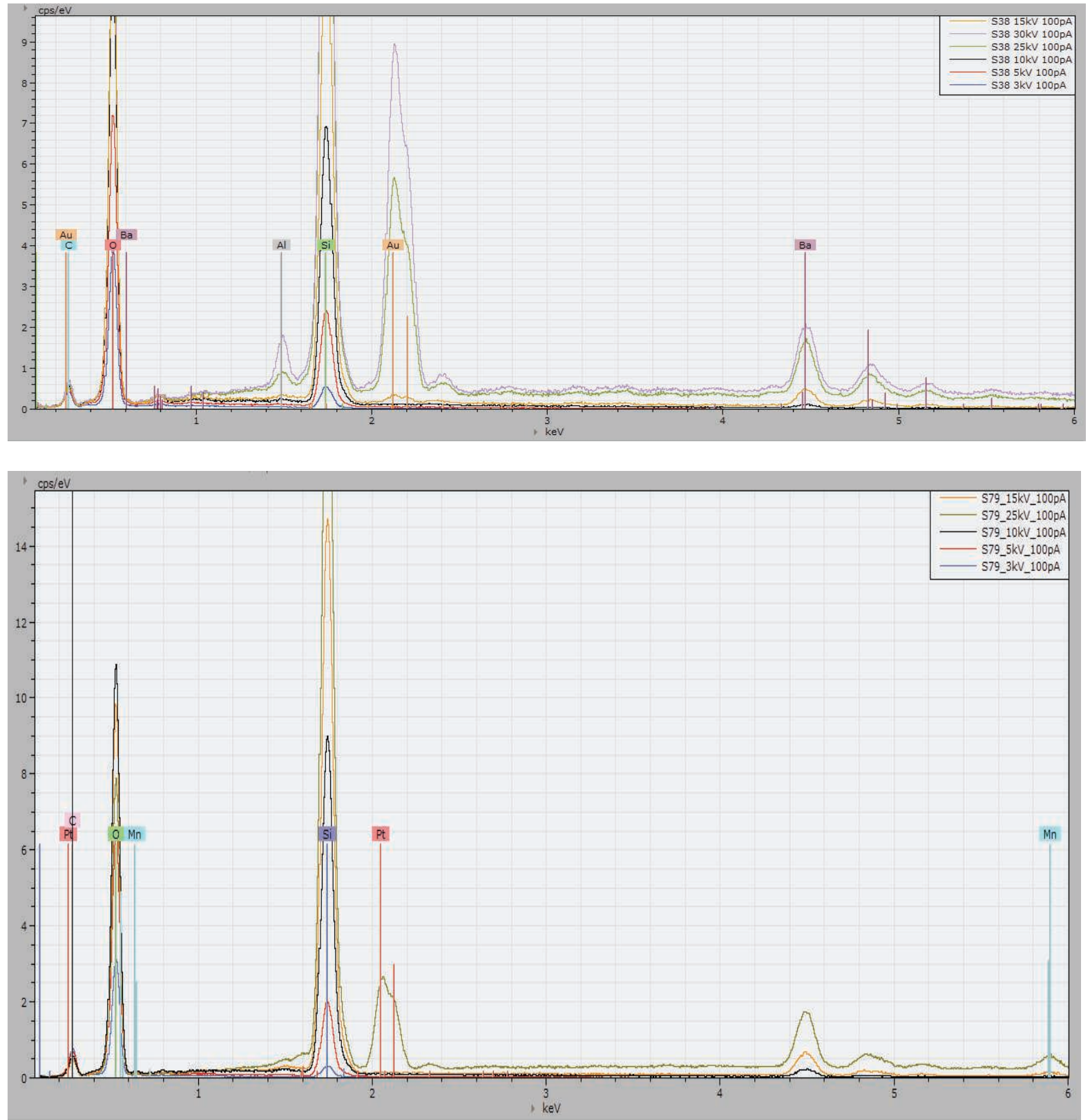

Abb. 8: Spektren von $\mathrm{pH}$-sensitiven Glasschichten bei unterschiedlichen Anregungsspannungen (o.: Ableitelektrode: Gold, 1800 s Abscheidezeit pH-Glas, u.: Ableitelektrode Platin, 3600 s Abscheidezeit pH-Glas)

$\mu$-RFA-Analysen beschrieben. Begründet durch die Schichtstruktur und das undefinierte Volumen, woraus die auswertbaren Signale generiert werden, sind keine quantitativen Analysen möglich. Dennoch bieten EDX-Analysen wertvolle Hinweise auf Schichtqualitäten und Schichtdicke. Durch die Verwendung eines Doppeldetektorsystems Quantax 400 D mit zwei XFlash ${ }^{\circledR}$-Detektoren der Fa. Bruker Nano $\mathrm{GmbH}$, Berlin an einem Rastermikroskop und es können Informationen aus unterschiedlichen Tiefen des zu untersuchenden Materials generiert werden. In Abbildung 8 sind zwei Spektrenserien mit unterschiedlichen Anregungsspannungen dargestellt. Es handelt sich um ein pH-Glas auf einer Goldableitelektrode (Abscheidedauer $1800 \mathrm{~s}$ ) und ein $\mathrm{pH}$-Glas auf einer Platinableitelektrode (Abscheidedauer 3600 s). Während man das Silizium des $\mathrm{pH}$ Glases in beiden Spektren bereits bei Anre- 
gungsspannungen von $3 \mathrm{kV}$ detektiert, registriert man erste Signale des darunterliegenden Goldes bei $15 \mathrm{kV}$ bzw. des darunterliegenden Platins bei doppelter Abscheidedauer erst bei 25 kV Anregungsspannung. Mit dieser Methode können Fehlstellen in der sensitiven Glasschicht sofort zugeordnet und verifiziert werden.

\section{Elektrochemische Charakterisierungen}

Für die elektrochemischen Untersuchungen und Langzeitmessungen erhielten die Sensorstrukturen, wie in Abbildung 9 dargestellt, hermetisch dichte Verkappungen.
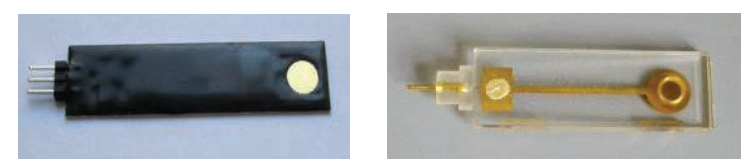

Abb. 9: $\quad$ hermetisch dicht verkappte Sensorstrukturen für Langzeituntersuchungen

Neben der elektrochemischen Impedanzspektroskopie kamen Untersuchungen zur Bestimmung des Sensorverhaltens, i.d.R. Sensorsteilheit, Sensordrift, Reproduzierbarkeit und Querempfindlichkeiten zum Einsatz. Verwendung hierfür fanden Multiparametermesssysteme der Fa. Sensortechnik Meinsberg $\mathrm{GmbH}$, Waldheim.

Die Sensorsysteme weisen i.d.R. Steilheiten von $40-45 \mathrm{mV} / \mathrm{pH}$ in einem $\mathrm{pH}$-Bereich von 2 - $10 \mathrm{pH}$ auf. In Abbildung 10 sind die Reproduzierbarkeitsmessungen eines $\mathrm{pH}$-Sensors in Dünnschichttechnik über 15 Tage dargestellt. Die Messungen fanden in NBS-Puffern und bei einer Temperatur von $25^{\circ} \mathrm{C}$ statt.

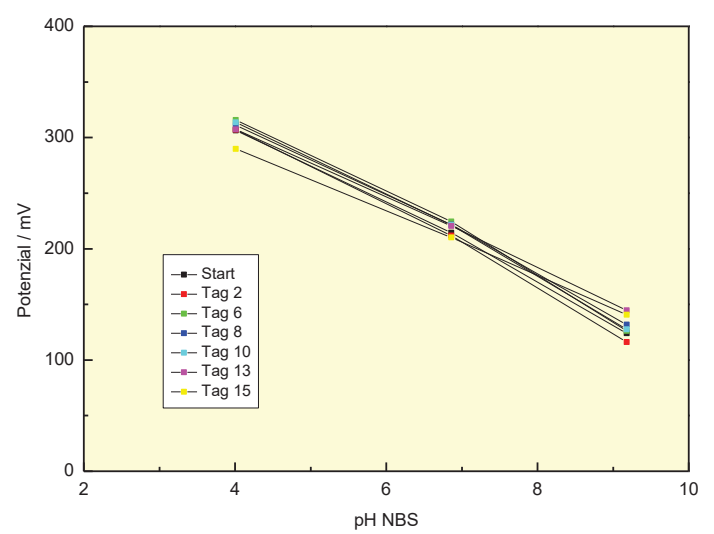

Abb. 10: Reproduzierbarkeit der Sensorsteilheit über einen Zeitraum von 15 Tagen

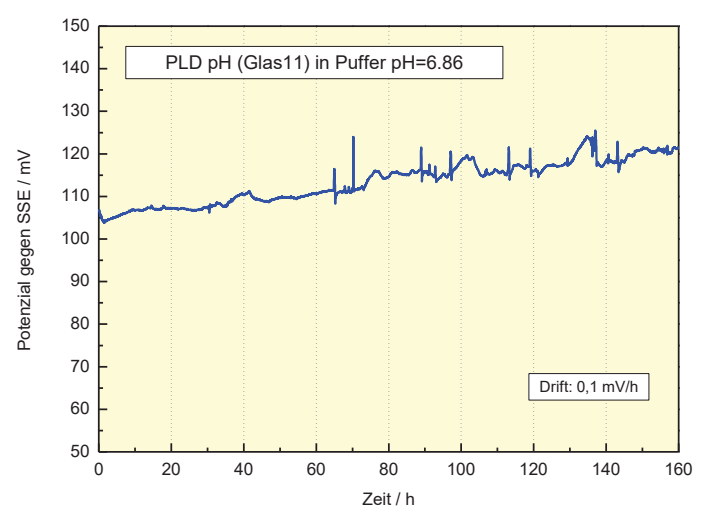

Abb. 11: Sensordrift eines Dünnschicht-pHSensors (Potenzialdrift: 0,1 $\mathrm{mV} / \mathrm{h}$ )

Ein wesentliches Kriterium für die Sensorstabilität stellt die Sensordrift dar. Die in Abbildung 11 dargestellte Kettenspannung der Dünnschichtelektrode wurde gegen eine konventionelle $\mathrm{Ag} / \mathrm{AgCl}$-Referenzelektrode mit gesättigtem $\mathrm{KCl}$-Innenelektrolyt bestimmt.

\section{Zusammenfassung}

Der vorliegende Beitrag beschreibt die Möglichkeiten der PLD-Technologie, insbesondere technologisch schwer beherrschbare, sensitive Gläser (z.B. sich nah an der Entmischungsgrenze befindliche redoxsensitive Gläser) zu homogenen, dichten Schichten amorph zu applizieren.

\section{Literaturnachweis}

[1] K. Ahlborn, F. Gerlach, W. Vonau, International Journal on Advances in Systems and Measurements, issn 1942-261x, vol.10, no. $1 \& 2,2017$, p. 56-63

[2] W. Vonau, F. Gerlach, K. Ahlborn, PLD as possible tool for the fabrication of chemosensors based on amorphous membranes, The 7th International Conference on Sensor Device Technologies and Applications 24.-28.07.2016, Nice, France

[3] B.B. He, U. Preckwinkel and K.L. Smith, Advances in X-ray Analysis, Vol.43, p. 273 - 280

[4] G. Eisenmann, Glass Electrodes for Hydrogen and other Cations. New York: Marcel Dekker, 1967

[5] B.P. Nikolsky et al., Patent US 3773642

[6] F. Gerlach et al., Sensor \& Transducers, 2015, 184,39

\section{Danksagung}

Diese wissenschaftlichen Arbeiten wurden teilweise über die AiF im Rahmen des Programms zur Förderung der Industriellen Gemeinschaftsforschung (IGF) vom Bundesministerium für Wirtschaft und Energie aufgrund eines Beschlusses des Deutschen Bundestages unter dem Förderkennzeichen 18665 BG der Forschungsvereinigung "Technik und Glas" gefördert. 